\title{
Childhood thyroid function, body composition and cardiovascular function
}

\author{
Mirjana Barjaktarovic 1,2,3, Tim I M Korevaar1,2,3, Romy Gaillard ${ }^{1,4}$, \\ Yolanda B de Rijke ${ }^{2,5}$, Theo J Visser ${ }^{2,3}$, Vincent W V Jaddoe ${ }^{1,4,6}$ and Robin P Peeters ${ }^{2,3}$ \\ ${ }^{1}$ The Generation R Study Group, ${ }^{2}$ Department of Internal Medicine, ${ }^{3}$ Rotterdam Thyroid Center and ${ }^{4}$ Department of \\ Epidemiology, Erasmus Medical Center, Rotterdam, The Netherlands, ${ }^{5}$ Department of Clinical Chemistry and \\ ${ }^{6}$ Department of Pediatrics, Erasmus Medical Center, Sophia Children's Hospital, Rotterdam, The Netherlands
}

Correspondence should be addressed to R P Peeters

Email

r.peeters@erasmusmc.nl

\begin{abstract}
Objective: The cardiovascular system is a known target for thyroid hormone. Early-life cardiovascular alterations may lead to a higher risk of cardiovascular disease in adulthood. Little is known about the effects of thyroid hormone on cardiovascular function during childhood, including the role of body composition in this association.

Design: Population-based prospective cohort of children ( $n=4251$, median age 6 years, 95\% range: $5.7-8.0$ years). Methods: Thyroid-stimulating hormone (TSH) and free thyroxine (FT4) concentrations were measured to assess thyroid function. Left ventricular (LV) mass was assessed with echocardiography. Arterial stiffness was assessed with carotidfemoral pulse wave velocity (CFPWV). Systolic and diastolic blood pressure (BP) was measured. Body composition was assessed by dual-energy $\mathrm{X}$-ray absorptiometry scan.

Results: FT4 was inversely associated with LV mass $(P=0.002)$, and with lean body mass $(P<0.0001)$. The association of FT4 with LV mass was partially mediated through variability in lean body mass ( $55 \%$ mediated effect). TSH was inversely associated with LV mass $(P=0.010)$, predominantly in boys. TSH was positively associated with systolic and diastolic BP (both $P<0.001)$. FT4 was positively associated with CFPWV and diastolic BP $(P<0.0001, P=0.008$, respectively), and the latter association attenuated after adjustment for CFPWV.

Conclusions: At the age of 6 years, higher FT4 is associated with lower LV mass (partially through effects on lean body mass) and with higher arterial stiffness, which may lead to higher BP. Our data also suggest different mechanisms via which TSH and FT4 are associated with cardiovascular function during early childhood.
\end{abstract}

\section{Introduction}

Cardiovascular disease is the leading cause of morbidity and mortality worldwide (1). Structural and functional cardiac parameters, as well as BP, track from childhood to adulthood $(2,3,4,5,6)$, suggesting that early-life metabolic and physiological alterations may lead to an adaptive response of the cardiovascular system. Consequently, the risk of developing cardiovascular disease in adult life may increase (7) and the determinants of childhood cardiac structure and function could have predictive value for clinical events in adulthood. Childhood cardiovascular structure and function are
() 2017 European Society of Endocrinology Printed in Great Britain mainly determined by changes in the body size that occur due to somatic growth (8); however, little is known about other determinants.

The cardiovascular system is a well-known target for thyroid hormone (TH), as is exhibited by profound effects of TH on systemic vascular resistance, contractility, heart rate, blood volume and cardiac mass (9). Different types of evidence also support a role for TH in the regulation of early cardiac function and growth $(10,11)$. During fetal and postnatal life, cardiomyocytes express $\mathrm{TH}$ receptors (TR $\alpha$ and TR $\beta$ ) (12) and the transcription of genes 
encoding contractile proteins of myosin heavy chains is TH dependent (13).

Although the role of thyroid function in the regulation of cardiovascular system has been extensively studied in adults, data about the effects of variation in thyroid function on cardiovascular development and function during childhood are scarce. Furthermore, there is a lack of data on the effects of thyroid function on body composition and its potential role in the association of $\mathrm{TH}$ with cardiovascular system in the pediatric population. Therefore, we aimed to study the association of childhood thyroid function with cardiovascular structure and function and body composition measurements.

\section{Subjects and methods}

\section{Study population}

This study was embedded in Generation R, a populationbased prospective cohort from early fetal life onwards in Rotterdam, The Netherlands (14). The study was designed to identify early environmental and genetic causes leading to normal and abnormal growth, development and health during fetal life and childhood (14). All children were born between 2002 and 2006 (14). In total, 6690 children (median age: 6 years, 95\% range: 5.78.0 years) attended the research center for the follow-up measurements. Successful serum sampling was performed in 4593 children and thyroid-stimulating hormone (TSH) and free thyroxine (FT4) concentrations were determined in 4306 serum samples. Children with thyroid/chronic disease and thyroid (interfering) medication, cardiac abnormalities or with missing data on cardiac ultrasound ( $n=23, n=32, n=182$, respectively) were excluded from the analysis (Supplementary Fig. 1, see section on supplementary data given at the end of this article).

\section{Ethics approval}

The general design, all research aims and the specific measurements in the Generation $\mathrm{R}$ study have been approved by the Medical Ethical Committee of the Erasmus Medical Center, Rotterdam. Written informed consent was obtained from all participants.

\section{Thyroid measurements}

Serum samples were obtained at the time of the visit to the research center. Plain tubes were centrifuged and serum was stored at $-80^{\circ} \mathrm{C}$. TSH and FT4 concentrations were determined using an electrochemiluminescence immunoassay on the Cobas e601 immunoanalyzer (Roche Diagnostics). The intra-assay and interassay coefficients of variation were $1.1-3.0 \%$ for TSH at a range of $0.04-0.4 \mathrm{U} / \mathrm{L}$ and $1.6-5.0 \%$ for FT4 at a range of $1.6-24.1 \mathrm{pmol} / \mathrm{L}$.

\section{Cardiovascular measurements}

M-mode echocardiographic measurements were performed using the ATL-Philips Model HDI 5000 (Seattle, WA, USA) or the Logiq E9 (GE Medical Systems, Wauwatosa, WI, USA) device $(15,16)$. Experienced sonographers performed the measurements and were supervised by a pediatric cardiologist. To minimize the inter-observer differences, quality checks were frequently carried out and feedback was provided regularly. To assess reproducibility of echocardiographic measurements, the intraobserver intraclass correlation coefficient were calculated for LAD, AOD, interventricular end-diastolic septum thickness (IVSD), left ventricular diastolic diameter (LVDD) and left ventricular posterior wall thickness (LVPWD) in 28 subjects (median age: 7.5 years, interquartile range 3.0-11.0) and varied between 0.91-0.99 and 0.78-0.96, respectively (17). Missing echocardiograms were random, and mainly due to participant circumstances or unavailability of equipment or sonographer. Aortic root diameter (AOD), LVDD, LVPWD and IVSD were measured and fractional shortening (FS) and LV mass were calculated; LV mass was computed using the formula by Devereux et al: LV mass $=0.80 \times 1.04$ ((IVSTD+LVEDD + LVPWTD) 3 - (LVEDD)3) + 0.6 (18).

Carotid-femoral pulse wave velocity (CFPWV), the reference method to assess arterial stiffness (19), was measured in a supine position using the Automatic Complior SP Device (Complior; Artech Medical, Pantin, France). CFPWV was calculated as a ratio of the distance traveled by the pulse wave and the time delay between the upstroke of carotid and femoral waveforms. The mean of at least 10 consecutive pressure waveforms was used in order to cover a complete respiratory cycle. CFPWV can be measured reliably, with good reproducibility, in large pediatric population-based cohorts (20).

Systolic blood pressure (BP) and diastolic BP were measured at the right brachial artery in a supine position, four times with one-minute intervals. The automatic sphygmomanometer Datascope Accutorr Plus (Paramus, New Jersey, USA) was used (21). The mean value was calculated using the last three blood pressure measurements. Pulse pressure was calculated as the difference between mean systolic and mean diastolic 
pressure. In this study high BP was defined as the highest 5 percentiles of the study population (22).

\section{Anthropometric measurements}

Height and weight were measured without shoes and heavy clothing and were used to determine body surface area (BSA), calculated according to the Haycock formula $(23,24)$. Dual-energy X-ray absorptiometry scan (iDXA, General Electrics - Lunar, 2008, Madison, WI, USA) was performed to assess the lean and fat mass of the body composition; subsequently, lean mass and fat mass indices were determined according to formula: lean mass index =lean mass $/$ height $^{2}$ and fat mass index =fat mass $/$ height $^{2}(25)$.

\section{Covariates}

Information on ethnicity was obtained through questionnaires and was classified by the countries of birth of the parents, according to the classification of Statistics Netherlands (14). Information on the educational level of the mother was used as a proxy for socioeconomic status and was obtained through questionnaires, as well as information about maternal smoking during pregnancy (14).

\section{Statistical analysis}

We investigated the associations of TSH and FT4 with mean LV mass, AOD, FS, lean mass, fat mass, CFPWV, systolic and diastolic BP using multiple linear regression analyses, utilizing restricted cubic splines with three knots to account for possible non-linear associations. Multiple logistic regression models were used to assess the association of TSH with the risk of high BP. All model covariates were selected based on biological plausibility, change of the effect estimate of interest or residual variability of the model. The analyses were adjusted for sex, age, ethnicity and maternal educational level. Maternal smoking during pregnancy did not affect the estimates and was not included in the model. For the models of cardiac ultrasound measurements, sensitivity analyses were performed to examine the potential effect modification by BP level. For the BP models, analyses were performed to examine the potential mediation effect of arterial stiffness, as well as to test the effect modification by BMI. Multivariable associations were graphically depicted by plots (main manuscript) and $\beta$ estimates with 95\% confidence intervals are shown in Supplementary Table 2. We tested for effect modification with sex by introducing a product interaction term of TSH or FT4 with sex to the model. A $P$ value cutoff of $<0.15$ was considered for quantification of the effect difference by subsequent stratification of the association.

We accounted for the high number of statistical tests (39 in total) by controlling the false discovery rate using the fdrtool package (26). This method allows for tailored identification of the expected proportion of false-positive results among all rejected null hypothesis. We identified that a $q$-value of 0.055 (i.e. the cutoff for a $5.5 \%$ chance of a type I error) was similar to a $P$ value of 0.05 . Therefore, a $P$ value threshold of $<0.05$ was considered for statistical significance.

As BSA explains a large percentage of the variability in cardiac size (8), BSA-adjusted SDS for AOD and LV mass were constructed for analyses. Height-adjusted SDS for BP and CFPWV were constructed for analyses. All SDS were constructed using Generalized Additive Models for Location, Size and Shape (GAMLSS).

Since $\mathrm{TH}$ regulates body composition, which is also an important determinant of LV mass, the association of thyroid function with cardiac ultrasound measurements could be mediated via changes in body composition $(27,28)$. To examine the mediating role of BSA in the association of FT4 with LV mass, we analyzed the direct and indirect mediation effects of BSA by performing mediation analysis, using the approach described previously by Imai et al. (29). To further examine the mediating effects of specific body composition components in the association of FT4 with LV mass, we performed similar analyses with the lean mass index as a potential mediator.

For covariates with missing data, multiple imputation according to Markov Chain Monte Carlo method was used (30). The percentage of missing data was $2.7 \%$ for ethnicity and $14.8 \%$ for maternal educational level variables. Five imputed data sets were created and pooled for analysis. Child ethnicity and maternal educational level were then added to the model. We added age, mean systolic and diastolic BP, TSH and FT4 concentrations and cardiac ultrasound measurements as prediction variables only. No statistically significant differences in descriptive statistics were found between the original and imputed datasets. Statistical analyses were performed using Statistical Package of Social Sciences, version 21.0 for Windows (SPSS) and R statistical software, version 3.2.0 (package rms, mediation, GAMLSS and fdrtool). 
Table 1 Descriptives of the study population.

\begin{tabular}{l} 
Characteristic \\
\hline TSH, median (95\% range), $\mathrm{mU} / \mathrm{L}$ \\
FT4, median ( $95 \%$ range), pmol/L \\
Age, median ( $95 \%$ range), years \\
BMI, mean, (s.D.), kg/m² \\
BSA (s.D.), $\mathrm{m}^{2}$ \\
Lean mass index, median (95\% range), \\
kg/m ${ }^{2}$ \\
Fat mass index, median (95\% range), \\
kg/m ${ }^{2}$ \\
Blood pressure, median (95\% range), \\
mmHg \\
Systolic \\
Diastolic \\
Pulse pressure \\
Carotid-femoral pulse wave velocity, \\
median, ( $95 \%$ range), m/s \\
Aortic root diameter, mean, (s.D.), mm \\
Left ventricular mass, mean, (s.D.), g \\
Fractional shortening, mean (s.D.), $\%$ \\
Ethnicity, $n$ (\%) \\
Dutch \\
Moroccan \\
Turkish \\
Surinamese \\
Other-Western \\
Other-Non-Western \\
Child sex, $n$ (\%) \\
Male \\
Female \\
Maternal education, $n$ (\%) \\
No education/primary \\
Secondary \\
Higher \\
\hline
\end{tabular}

\begin{tabular}{c}
\hline Value \\
\hline $2.29(0.87-5.20)$ \\
$16.8(13.8-20.7)$ \\
$6.0(5.7-8.0)$ \\
$16.2(1.7)$ \\
$0.9(0.1)$ \\
$1.14(0.98-1.33)$ \\
$0.37(0.24-0.79)$ \\
\\
$102(88-120)$ \\
$60(48-74)$ \\
$42(30-56)$ \\
$5.39(4.08-7.53)$ \\
$19.3(1.8)$ \\
$53.8(11.7)$ \\
$35.3(4.5)$ \\
$2439(57.6)$ \\
$266(6.3)$ \\
$305(7.2)$ \\
$308(7.3)$ \\
$339(8.0)$ \\
$581(13.7)$ \\
$2199(51.5)$ \\
$2071(48.5)$ \\
$196(4.6)$ \\
$1692(39.9)$ \\
$2350(55.5)$ \\
\end{tabular}

\section{Results}

After exclusions, the final study population consisted of 4251 children (Supplementary Fig. 1), descriptives of which are shown in Table 1 . There were no differences between the participants with or without $(n=182)$ available cardiac ultrasound data (Supplementary Table 3) with the exception of FT4 concentrations (median 16.8 vs $16.4, P=0.033)$. There was no difference between participants with or without available data on CFPVW (Supplementary Table 4) or BP (data not shown).

\section{The association of thyroid function with cardiac structure and function}

There was an inverse linear association of both TSH and FT4 concentrations with $\mathrm{LV}$ mass $(P=0.010$ and $P=0.002$, respectively; Fig. $1 \mathrm{~A}$ and $\mathrm{B})$, which remained same after additional adjustment for BP. TSH and FT4 concentrations were not associated with AOD or FS (Fig. 1C, D, E and F).
After addition of a product interaction term to the model (TSH*sex, $P=0.09$ ), we stratified the association of TSH with LV mass by sex (Supplementary Fig. 2). The association of TSH with LV mass was present in boys and not in girls ( $P=0.004$ and $P=0.46$, respectively; Supplementary Fig. 2). There were no sex-specific differences in the association of FT4 concentration with LV mass or in the associations of TSH and FT4 with AOD or FS.

\section{The role of body composition in the association of thyroid function with left ventricular mass}

We observed an inverse linear association of FT4 with BSA (beta coefficient $=-0.002,95 \%$ confidence interval: (-0.004, -0.001), $P$ value $<0.001$, data not shown), and we subsequently investigated if the association of FT4 with LV mass could be mediated by BSA. The mediation analysis showed that $26 \%$ of the effect of FT4 on LV mass was mediated via changes in BSA (Table 2). As BSA roughly reflects the variability in lean mass and fat mass, and TH may differentially affect these body composition segments (31), we further investigated the association of thyroid function with lean mass and fat mass. There was an inverse linear association of FT4 with lean mass (Fig. 2B, $P<0.0001$ ), whereas FT4 was not associated with fat mass (Fig. 2D, $P=0.92$ ). TSH was not associated with lean mass or fat mass (Fig. 2A and C, $P=0.76, P=0.09$, respectively).

To specifically study the mediating role of the particular body composition components in the association of FT4 with LV mass, we performed a mediation analysis with the lean mass index as a potential mediator. This analysis showed that $55 \%$ of the association was mediated through changes in lean mass (Table 3 ).

\section{The association of thyroid function with CFPWV and blood pressure}

TSH was not associated with CFPWV ( $P=0.59$, Fig. 3A) whereas there was a positive linear association of FT4 with CFPWV $(P<0.0001$, Fig. 3B).

There was a positive linear association of TSH with systolic and diastolic BP $(P<0.0001$ and $P=0.0001$ respectively; Fig. 4A and $\mathrm{C}$ ). The association of TSH with systolic BP was more prominent in girls, and girls with higher TSH concentration had a higher risk of high $\mathrm{BP}(\mathrm{OR}$ 1.92-2.12 depending on the TSH cut-off; Supplementary Fig. 3). FT4 was not associated with systolic BP, whereas there was a positive linear association of FT4 with diastolic 
A

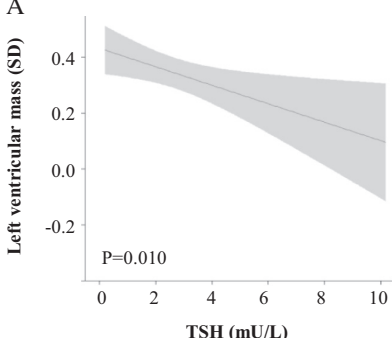

$\mathrm{C}$
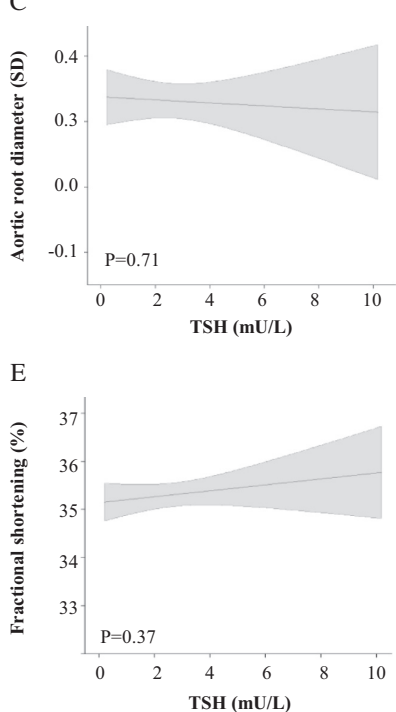

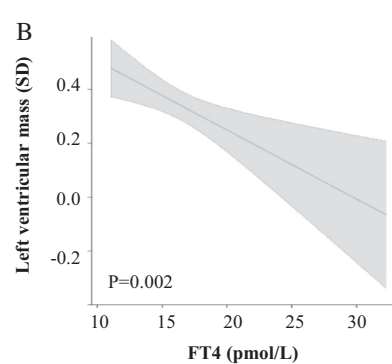

D

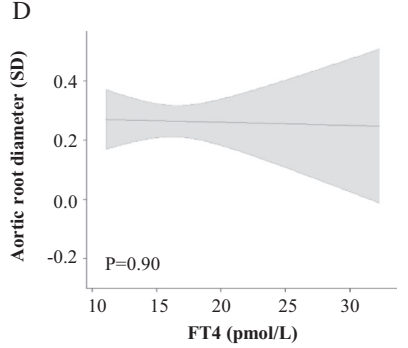

F

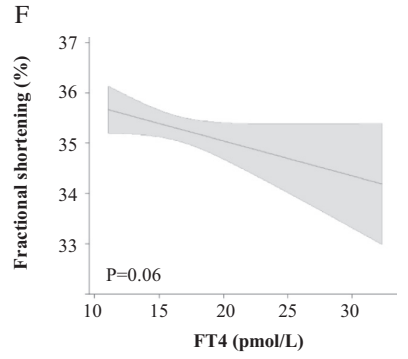

\section{Figure 1}

Plots show the linear regression models for TSH, FT4 and cardiac ultrasound measurements, as predicted mean with $95 \%$ confidence interval. Analyses were adjusted for ethnicity, age, sex and maternal educational level.

$\mathrm{BP}(P=0.21$ and $P=0.008$, respectively; Fig. $4 \mathrm{~B}$ and $\mathrm{D})$. There was a positive linear association of CFPWV with BP (beta coefficient $0.15 \pm 0.02, P<0.001$ and $0.18 \pm 0.02, P<0.001$ for systolic and diastolic BP, respectively, data not shown). After adding CFPWV to the model, the association of FT4 with diastolic BP attenuated (beta coefficient decreased $30 \%$ (from $0.022 \pm 0.009$ to $0.016 \pm 0.009, P=0.07$, data not shown.) There was no association of TSH or FT4 with pulse pressure (data not shown).

Table 2 BSA as a mediator in the association of FT4 with LV mass.

\begin{tabular}{|c|c|c|c|}
\hline Mediator: BSA & Estimate & $95 \%$ confidence interval & $P$ value \\
\hline Mediated effect & -0.164 & $-0.275,-0.064$ & $<0.001$ \\
\hline Direct effect & -0.465 & $-0.611,-0.306$ & $<0.001$ \\
\hline Total effect & -0.629 & $-0.830,-0.453$ & $<0.001$ \\
\hline $\begin{array}{l}\text { Percentage of } \\
\text { mediated effect }\end{array}$ & $26 \%$ & & \\
\hline
\end{tabular}

\section{Discussion}

Early-life determinants may shape the development of the cardiovascular system and influence subsequent predisposition for cardiovascular disease in adult life. In the current study, we show that thyroid function is a determinant of LV mass, and this effect is partially mediated by the effects of FT4 on lean body mass. We also demonstrate a positive association of TSH with BP and a positive association of FT4 with diastolic BP that was dependent on the differences in arterial stiffness, suggesting that TSH and FT4 are associated with BP via different mechanisms.

Little is known about the effects of THs on cardiac structure and function at a young age, and to our knowledge, no study has investigated the association of thyroid function with LV mass in children. In adults, population-based studies do not observe an association of TSH concentrations with LV mass $(32,33,34)$, whereas a higher LV mass has been described in individuals with subclinical and/or overt hyperthyroidism $(35,36$, 37), although a longitudinal study showed no effect of subclinical hyperthyroidism on the progression of cardiac hypertrophy in adults (38). One study in hypertensive individuals reports an inverse linear association of TSH concentration with LV mass (39). In the current study, there was an inverse linear association of TSH concentration with LV mass in children. Importantly, various differences between adult and childhood factors need to be taken into account when interpreting these associations. In children, the changes in LV mass are predominantly determined by changes in body size (8), and the influence of pathological conditions on the cardiovascular system is much lower than in adults. Factors contributing to a chronically high cardiac load likely underlie the association of thyroid function with LV mass in hyperthyroid and hypertensive state during adulthood $(35,36,39)$, whereas during childhood, LV mass is essentially determined by growth (8). This is in line with our results showing no effect of additional adjustment for BP. An alternative explanation for the effects of TSH could potentially be the underling effects of thyrotropin-releasing hormone (TRH) on the cardiovascular system, as studies in animals demonstrate a positive association of the TRH concentration LV mass $(40,41)$. Furthermore, in our study, higher TSH was associated with lower LV mass only in boys. Interestingly, studies in adults report no sex-specific differences in the association of thyroid function with LV mass (32, 33, 34, 39 ). Hence, these differences are more likely to reflect a sex-specific diversity in the rate of both somatic growth as well as heart growth, as LV mass is higher in boys than 

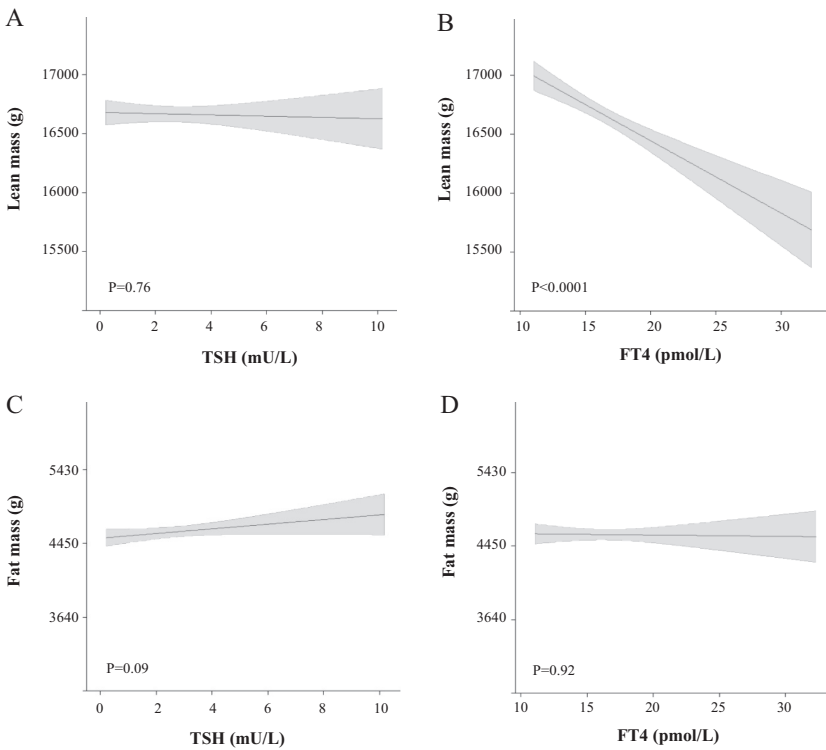

\section{Figure 2}

Plots show the linear regression models for TSH and FT4 with lean mass and fat mass, as predicted mean with $95 \%$ confidence interval. Analyses were adjusted for sex, ethnicity, age, height and maternal educational level.

in girls already in the prepubertal period (42). This is in line with the higher TSH concentrations in boys in the Generation R cohort (unpublished data), suggesting a potential for stronger effects of TSH in boys.

Surprisingly, not only higher TSH, but also higher FT4 was associated with lower LV mass. Most of the previous studies on thyroid function and cardiac structure did not examine the association of FT4 with LV mass (32, 33, 34, 35) and only focused on TSH as a measurement of thyroid function. A positive association of FT4 with LV mass has been reported in euthyroid hypertensive adults (39), most likely due to cardiovascular adaptations induced by chronic hypertension and relatively high BMI of the study participants. In our study, the association of FT4 with LV mass was partially mediated via the effects of FT4 on the lean body mass but not fat mass. Similar to a previous study,

Table 3 Lean body mass as a mediator in the association of of FT4 with LV mass.

\begin{tabular}{|c|c|c|c|}
\hline $\begin{array}{l}\text { Mediator: leanbody } \\
\text { mass index }\end{array}$ & Estimate & $\begin{array}{l}95 \% \text { confidence } \\
\text { interval }\end{array}$ & $P$ value \\
\hline Mediated effect & -0.339 & $-0.423,-0.249$ & $<0.001$ \\
\hline Direct effect & -0.282 & $-0.465,-0.098$ & $<0.001$ \\
\hline Total effect & -0.620 & $-0.810,-0.421$ & $<0.001$ \\
\hline $\begin{array}{l}\text { Percentage of } \\
\text { mediated effect }\end{array}$ & $55 \%$ & & \\
\hline
\end{tabular}

higher FT4 was associated with lower lean mass (31), and we subsequently identified that this effect may mediate approximately $55 \%$ of the association of FT4 with LV mass. This is in line with the notion that lean mass is a much stronger determinant of LV mass than fat mass in children $(27,43)$. Taken together, our results suggest that the association of thyroid function with LV mass is a reflection of different biological mechanisms through which TSH and FT4 are associated with LV mass, which could explain a similar direction of these associations. The inverse association of FT4 with LV mass in our study suggests that children with lower FT4 are more likely to have higher LV mass at this age, and it remains to be determined if these individuals have a higher tendency toward LV hypertrophy in later life (2). The underlying mechanism of this association remains unclear, but it is likely that in the healthy population of a young age, we examined the developmental influence of $\mathrm{TH}$ on cardiac structure and function.

We did not observe an association of thyroid function with fractional shortening and aortic root diameter, which is in line with previous reports in adults $(33,34)$. This lack of association suggests that, at the age of 6 years, the effects of thyroid function are predominant on the cardiac structure and/or growth, as represented by changes in the LV mass. Large differences in thyroid function that could be caused by underlying thyroid disease are practically absent in this young population. As a consequence, any association of thyroid function with cardiac functional parameters will probably be less pronounced. As LV mass tracks from childhood to adulthood $(2,3)$, the observed associations might have implications for cardiac function in the future. However, future studies are needed to further study such long-term effects.

In line with previous reports $(44,45)$, we show that higher TSH is associated with higher BP. A positive association of TSH and BP was reported earlier in obese children as well, but here, the association of TSH with BP may have been amplified because obesity has been shown to affect both TSH and BP levels $(46,47)$. However, we did not find any effect modification by BMI. It has been speculated that this association might be occurring through common genetic factors that are associated with both thyroid function and BP (48).

A novel finding of this study is that FT4 is associated with diastolic BP via differences in arterial stiffness. The mechanism underlying this association is likely to be different from the association of TSH with BP, as in our study, CFPWV (a measure of arterial stiffness), was strongly associated with FT4 but not with TSH. Previous studies have also reported a positive association 

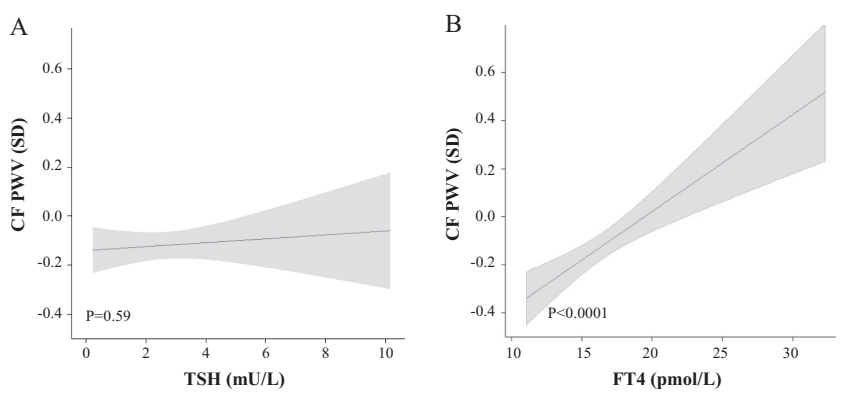

Figure 3

Plots show the logistic regression models for TSH/FT4 and carotid femoral pulse wave velocity, as predicted mean with $95 \%$ confidence interval. Analyses were adjusted for ethnicity, age, child sex and BMI.

of FT4 with arterial stiffness $(49,50)$ and speculated that FT4 modifies arterial elasticity (50). TH can initiate endothelial dysfunction $(51,52)$, which is an early sign of atherosclerosis (53) that underlies arterial stiffness. Taken together, this suggests that direct effects of TH on arterial tissue may create structural changes that could have consequences for BP levels already during childhood.

To our knowledge, this is the first population-based study that examined the association of thyroid function

(
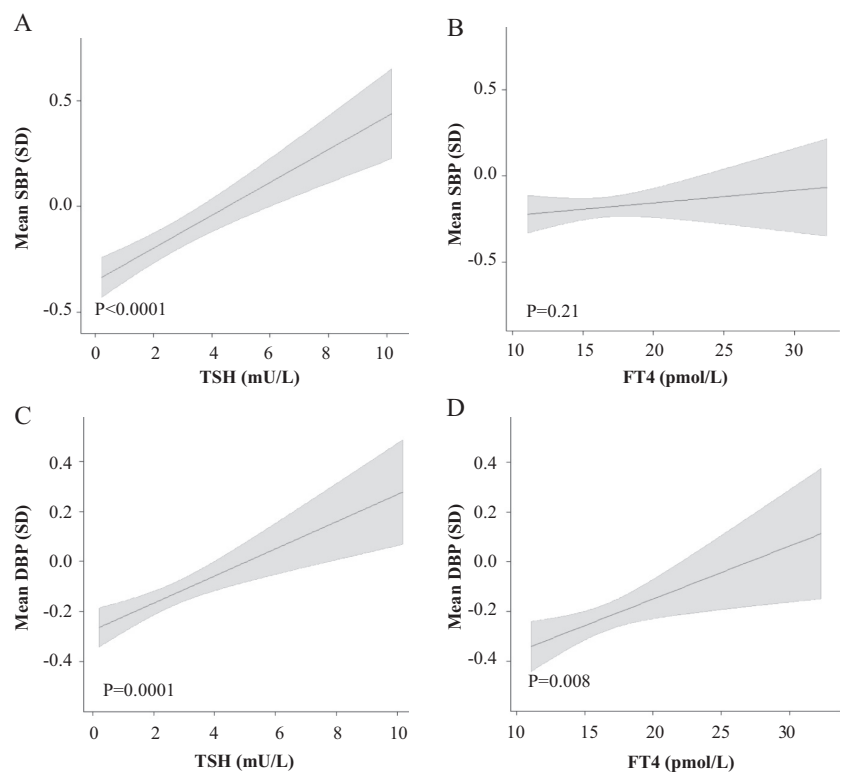

\section{Figure 4}

Plots show the linear regression models for TSH, FT4 and systolic/diastolic blood pressure, as predicted mean with $95 \%$ confidence interval. Analyses were adjusted for ethnicity, age, sex, maternal education level and BMI. with cardiovascular structural and functional parameters during childhood. We had detailed data available on thyroid function, cardiac ultrasound measurements, serial BP measurements, arterial stiffness, body composition assessment and potential confounding variables. The main limitation of this study is the cross-sectional study design, which does not allow for studying casual inference and does not impede residual confounding. Another limitation is the narrow age range of children (5.7-8.0 years), which limits the generalization of the results to older ages in childhood. Furthermore, in this study, we identified lean mass, and not fat mass, as a potential mediator of the effects of thyroid function on LV mass. The association of $\mathrm{TH}$ with fat mass is complex, potentially bidirectional and/or confounded by food intake/fasting (54). In adults, a positive association of FT4 with fat body mass has been described (31). In our study, however, the FT4 concentration was not associated with fat mass, rendering the possibility of mediation pathway through fat mass implausible.

In the current study, we translate the findings of the effects of thyroid function on cardiovascular growth and function from experimental studies to humans in the young age. We observed that thyroid function is associated with the measurement of cardiac growth (LV mass), in part via effects on lean body mass. We also observed that TSH and FT4 might have different underlying mechanisms in the association with BP, with a role of arterial stiffness in the latter case. Although these findings have relatively limited clinical implications, the observed associations designate the importance of thyroid function as an important cardiovascular determinant, as it might affect cardiovascular health during the lifespan. These results need further research to confirm the observations and investigate the association of thyroid function with longitudinal cardiac structure and function measurements during childhood, as well as the extent of arterial stiffness effect in the association of FT4 with BP.

\section{Supplementary data \\ This is linked to the online version of the paper at http://dx.doi.org/10.1530/} EJE-17-0369.

Declaration of interest

The authors declare that there is no conflict of interest that could be perceived as prejudicing the impartiality of the research reported.

\section{Funding}

This work was supported by a PhD grant from ERAWEB, a project funded by the European Commission (to M B) and by clinical fellowship from The Netherlands Organization for Health Research and Development (ZonMw), Project 90700412 (to R P P). The Generation R study is conducted by the 
Erasmus Medical Center (Rotterdam) in close collaboration with the School of Law and faculty of Social Sciences of the Erasmus University Rotterdam; the Municipal Health Service Rotterdam area, Rotterdam; the Rotterdam Homecare Foundation, Rotterdam and the Stichting Trombosedienst and Artsenlaboratorium Rijnmond, Rotterdam. The general design of the Generation R study is made possible by financial support from the Erasmus Medical Center, Rotterdam; the Erasmus University Rotterdam; The Netherlands Organization for Health Research and Development; The Netherlands Organization for Scientific Research; the Ministry of Health, Welfare and Sport; the Ministry of Youth and Families.

\section{Author contribution statement}

$M$ B performed data analyses and was involved in writing of the report. $R$ G, V W V and T J V contributed to the data analyses and writing of the report. Y B D R participated in the data collection and contributed to writing of the report. T I M K supervised analyses and contributed to writing of the report. R P P supervised analyses, contributed to writing of the report and directed the project.

\section{Acknowledgements}

The contribution of the endocrine laboratory technicians is highly appreciated. We gratefully acknowledge the contribution of children and parents, general practitioners, hospitals, midwives and pharmacies in Rotterdam.

\section{References}

1 Writing Group M, Mozaffarian D, Benjamin EJ, Go AS, Arnett DK, Blaha MJ, Cushman M, Das SR, de Ferranti S, Despres JP et al. Heart disease and stroke statistics-2016 update: a report from the American Heart Association. Circulation 2016133 e38-e360. (doi:10.1161/ CIR.0000000000000350)

2 Janz KF, Dawson JD \& Mahoney LT. Predicting heart growth during puberty: the Muscatine Study. Pediatrics 2000105 E63. (doi:10.1542/ peds.105.5.e63)

3 Schieken RM, Schwartz PF \& Goble MM. Tracking of left ventricular mass in children: race and sex comparisons: the MCV Twin Study. Medical College of Virginia. Circulation 199897 1901-1906. (doi:10.1161/01.CIR.97.19.1901)

4 Lam CSP, Xanthakis V, Sullivan LM, Lieb W, Aragam J, Redfield MM, Mitchell GF, Benjamin EJ \& Vasan RS. Aortic root remodeling over the adult life course longitudinal data from the framingham heart study. Circulation 2010122 884-890. (doi:10.1161/ CIRCULATIONAHA.110.937839)

5 Geelhoed JJ, Steegers EA, van Osch-Gevers L, Verburg BO, Hofman A, Witteman JC, van der Heijden AJ, Helbing WA \& Jaddoe VW. Cardiac structures track during the first 2 years of life and are associated with fetal growth and hemodynamics: the Generation R Study. American Heart Journal 2009158 71-77. (doi:10.1016/j.ahj.2009.04.018)

6 Chen X \& Wang Y. Tracking of blood pressure from childhood to adulthood: a systematic review and meta-regression analysis. Circulation 2008117 3171-3180. (doi:10.1161/ CIRCULATIONAHA.107.730366)

7 Geelhoed JJM \& Jaddoe VWV. Early influences on cardiovascular and renal development. European Journal of Epidemiology 201025 677-692. (doi:10.1007/s10654-010-9510-0)

8 Gutgesell HP \& Rembold CM. Growth of the human heart relative to body surface area. American Journal of Cardiology 199065 662-668. (doi:10.1016/0002-9149(90)91048-B)

9 Danzi S \& Klein I. Thyroid hormone and the cardiovascular system. Medical Clinics of North America 201296 257-268. (doi:10.1016/j. mcna.2012.01.006)
10 Li M, Iismaa SE, Naqvi N, Nicks A, Husain A \& Graham RM. Thyroid hormone action in postnatal heart development. Stem Cell Research 201413 582-591. (doi:10.1016/j.scr.2014.07.001)

11 Forhead AJ \& Fowden AL. Thyroid hormones in fetal growth and prepartum maturation. Journal of Endocrinology 2014221 R87-R103. (doi:10.1530/JOE-14-0025)

12 Wiersinga WM. The role of thyroid hormone nuclear receptors in the heart: evidence from pharmacological approaches. Heart Failure Reviews 201015 121-124. (doi:10.1007/s10741-008-9131-9)

13 Morkin E. Regulation of myosin heavy chain genes in the heart. Circulation 199387 1451-1460. (doi:10.1161/01.CIR.87.5.1451)

14 Jaddoe VW, van Duijn CM, Franco OH, van der Heijden AJ, van Iizendoorn MH, de Jongste JC, van der Lugt A, Mackenbach JP, Moll $\mathrm{HA}$, Raat $\mathrm{H}$ et al. The generation R study: design and cohort update 2012. European Journal of Epidemiology 201227 739-756. (doi:10.1007/ s10654-012-9735-1)

15 Lang RM, Bierig M, Devereux RB, Flachskampf FA, Foster E, Pellikka PA, Picard MH, Roman MJ, Seward J \& Shanewise J. Recommendations for chamber quantification. European Heart Journal-Cardiovascular Imaging 20067 79-108. (doi:10.1016/j. euje.2005.12.014)

16 Picard MH, Popp RL \& Weyman AE. Assessment of left ventricular function by echocardiography: a technique in evolution. Journal of the American Society of Echocardiography 200821 14-21. (doi:10.1016/j. echo.2007.11.007)

17 Geelhoed MJ, Snijders SP, Kleyburg-Linkers VE, Steegers EA, van Osch-Gevers L \& Jaddoe VW. Reliability of echocardiographic measurements of left cardiac structures in healthy children. Cardiology in the Young 200919 494-500. (doi:10.1017/S1047951109990862)

18 Devereux RB, Alonso DR, Lutas EM, Gottlieb GJ, Campo E, Sachs I $\&$ Reichek N. Echocardiographic assessment of left ventricular hypertrophy: comparison to necropsy findings. American Journal of Cardiology 198657 450-458. (doi:10.1016/0002-9149(86)90771-X)

19 Vlachopoulos C, Aznaouridis K \& Stefanadis C. Prediction of cardiovascular events and all-cause mortality with arterial stiffness: a systematic review and meta-analysis. Journal of the American College of Cardiology 201055 1318-1327. (doi:10.1016/j.jacc.2009.10.061)

20 Donald AE, Charakida M, Falaschetti E, Lawlor DA, Halcox JP, Golding J, Hingorani AD, Smith GD \& Deanfield JE. Determinants of vascular phenotype in a large childhood population: the Avon Longitudinal Study of Parents and Children (ALSPAC). European Heart Journal 201031 1502-1510. (doi:10.1093/eurheartj/ehq062)

21 Wong SN, Tz Sung RY \& Leung LC. Validation of three oscillometric blood pressure devices against auscultatory mercury sphygmomanometer in children. Blood Pressure Monitoring 200611 281-291. (doi:10.1097/01.mbp.0000209082.09623.b4)

22 American Academy of P. National high blood pressure education program working group on high blood pressure in children and adolescents. Pediatrics 2004114 iv-iv.

23 Haycock GB, Schwartz GJ \& Wisotsky DH. Geometric method for measuring body surface area: a height-weight formula validated in infants, children, and adults. Journal of Pediatrics 197893 62-66. (doi:10.1016/S0022-3476(78)80601-5)

24 Verbraecken J, Van de Heyning P, De Backer W \& Van Gaal L. Body surface area in normal-weight, overweight, and obese adults. A comparison study. Metabolism 200655 515-524. (doi:10.1016/j. metabol.2005.11.004)

25 Wells JCK \& Cole TJ. Adjustment of fat-free mass and fat mass for height in children aged 8 y. International Journal of Obesity and Related Metabolic Disorders 200226 947-952. (doi:10.1038/sj.ijo.0802027)

26 Benjamini Y \& Hochberg Y. Controlling the false discovery rate: a practical and powerful approach to multiple testing. Journal of the Royal Statistical Society $1995 \mathbf{5 7} 289-300$.

27 Daniels SR, Kimball TR, Morrison JA, Khoury P, Witt S \& Meyer RA. Effect of lean body mass, fat mass, blood pressure, and sexual maturation on left ventricular mass in children and adolescents. 
Statistical, biological, and clinical significance. Circulation 199592 3249-3254. (doi:10.1161/01.CIR.92.11.3249)

28 Knudsen N, Laurberg P, Rasmussen LB, Bülow I, Perrild H, Ovesen L \& Jørgensen T. Small differences in thyroid function may be important for body mass index and the occurrence of obesity in the population. Journal of Clinical Endocrinology and Metabolism 200590 4019-4024. (doi:10.1210/jc.2004-2225)

29 Imai K, Keele L \& Yamamoto T. Identification, inference and sensitivity analysis for causal mediation effects. Statistical Science 2010 25 51-71. (doi:10.1214/10-STS321)

30 Sterne JA, White IR, Carlin JB, Spratt M, Royston P, Kenward MG, Wood AM \& Carpenter JR. Multiple imputation for missing data in epidemiological and clinical research: potential and pitfalls. BMJ 2009 338 b2393. (doi:10.1136/bmj.b2393)

31 Roef G, Lapauw B, Goemaere S, Zmierczak HG, Toye K, Kaufman JM \& Taes Y. Body composition and metabolic parameters are associated with variation in thyroid hormone levels among euthyroid young men. European Journal of Endocrinology 2012167 719-726. (doi:10.1530/EJE-12-0447)

32 Iqbal A, Schirmer H, Lunde P, Figenschau Y, Rasmussen K \& Jorde R. Thyroid stimulating hormone and left ventricular function. Journal of Clinical Endocrinology and Metabolism 200792 3504-3510. (doi:10.1210/jc.2007-0727)

33 Pearce EN, Yang Q, Benjamin EJ, Aragam J \& Vasan RS. Thyroid function and left ventricular structure and function in the Framingham Heart Study. Thyroid 201020 369-373. (doi:10.1089/ thy.2009.0272)

34 Roef GL, Taes YE, Kaufman JM, Van Daele CM, De Buyzere ML, Gillebert TC \& Rietzschel ER. Thyroid hormone levels within reference range are associated with heart rate, cardiac structure, and function in middle-aged men and women. Thyroid 201323 947-954. (doi:10.1089/thy.2012.0471)

35 Dorr M, Wolff B, Robinson DM, John U, Ludemann J, Meng W, Felix SB \& Volzke H. The association of thyroid function with cardiac mass and left ventricular hypertrophy. Journal of Clinical Endocrinology and Metabolism 200590 673-677. (doi:10.1210/ jc.2004-1554)

36 Faber J \& Selmer C. Cardiovascular disease and thyroid function. Frontiers of Hormone Research $2014 \mathbf{4 3}$ 45-56.

37 Marcisz C, Jonderko G, Wroblewski T, Kurzawska G \& Mazur F. Left ventricular mass in patients with hyperthyroidism. Medical Science Monitor 200612 CR481-CR486.

38 Dorr M, Ittermann T, Aumann N, Obst A, Reffelmann T, Nauck M, Wallaschofski H, Felix SB \& Volzke H. Subclinical hyperthyroidism is not associated with progression of cardiac mass and development of left ventricular hypertrophy in middle-aged and older subjects: results from a 5-year follow-up. Clinical Endocrinology 201073 821-826. (doi:10.1111/j.1365-2265.2010.03882.x)

39 Iida M, Yamamoto M, Ishiguro Y, Yamazaki M, Honjo H \& Kamiya $\mathrm{K}$. Thyroid hormone within the normal range is associated with left ventricular mass in patients with hypertension. Journal of the American Society of Hypertension 20126 261-269. (doi:10.1016/j. jash.2012.04.002)

40 Schuman ML, Peres Diaz LS, Landa MS, Toblli JE, Cao G, Alvarez AL, Finkielman S, Pirola CJ \& Garcia SI. Thyrotropin-releasing hormone overexpression induces structural changes of the left ventricle in the normal rat heart. American Journal of Physiology-Heart and Circulatory Physiology 2014307 H1667-H1674. (doi:10.1152/ ajpheart.00494.2014)
41 Schuman ML, Landa MS, Toblli JE, Peres Diaz LS, Alvarez AL, Finkielman S, Paz L, Cao G, Pirola CJ \& Garcia SI. Cardiac thyrotropin-releasing hormone mediates left ventricular hypertrophy in spontaneously hypertensive rats. Hypertension 201157 103-109. (doi:10.1161/HYPERTENSIONAHA.110.161265)

42 de Simone G, Devereux RB, Daniels SR \& Meyer RA. Gender differences in left ventricular growth. Hypertension 199526 979-983. (doi:10.1161/01.HYP.26.6.979)

43 Gishti O, Gaillard R, Durmus B, Abrahamse M, van der Beek EM, Hofman A, Franco OH, de Jonge LL \& Jaddoe VW. BMI, total and abdominal fat distribution, and cardiovascular risk factors in schoolage children. Pediatric Research 201577 710-718.

44 Ittermann T, Thamm M, Wallaschofski H, Rettig R \& Volzke H. Serum thyroid-stimulating hormone levels are associated with blood pressure in children and adolescents. Journal of Clinical Endocrinology and Metabolism 201297 828-834. (doi:10.1210/jc.2011-2768)

45 Chen H, Xi Q, Zhang H, Song B, Liu X, Mao X, Li J, Shen H, Tang W, Zhang $\mathrm{J}$ et al. Investigation of thyroid function and blood pressure in school-aged subjects without overt thyroid disease. Endocrine 201241 122-129. (doi:10.1007/s12020-011-9517-7)

46 Minami Y, Takaya R, Takitani K, Ishiro M, Okasora K, Niegawa T \& Tamai H. Association of thyroid hormones with obesity and metabolic syndrome in Japanese children. Journal of Clinical Biochemistry and Nutrition 201557 121-128. (doi:10.3164/jcbn.15-24)

47 Aypak C, Turedi O, Yuce A \& Gorpelioglu S. Thyroid-stimulating hormone (TSH) level in nutritionally obese children and metabolic co-morbidity. Journal of Pediatric Endocrinology and Metabolism 201326 703-708.

48 Gumieniak O, Perlstein TS, Hopkins PN, Brown NJ, Murphey LJ, Jeunemaitre X, Hollenberg NK \& Williams GH. Thyroid function and blood pressure homeostasis in euthyroid subjects. Journal of Clinical Endocrinology and Metabolism 200489 3455-3461. (doi:10.1210/ jc.2003-032143)

49 Czarkowski M, Hilgertner L, Powalowski T \& Radomski D. The stiffness of the common carotid artery in patients with Graves' disease. International Angiology 200221 152-157.

50 Delitala AP, Orru M, Filigheddu F, Pilia MG, Delitala G, Ganau A, Saba PS, Decandia F, Scuteri A, Marongiu M et al. Serum free thyroxine levels are positively associated with arterial stiffness in the SardiNIA study. Clinical Endocrinology 201582 592-597. (doi:10.1111/ cen.12532)

51 Poplawska-Kita A, Siewko K, Telejko B, Modzelewska A, Mysliwiec J, Milewski R, Gorska M \& Szelachowska M. The changes in the endothelial function and haemostatic and inflammatory parameters in subclinical and overt hyperthyroidism. International Journal of Endocrinology 20132013 article ID 981638. (doi:10.1155/2013/981638)

52 Poplawska-Kita A, Szelachowska M, Modzelewska A, Siewko K, Dzieciol J, Klimiuk PA \& Gorska M. Endothelial dysfunction in Graves' disease. Advances in Medical Science 201358 31-37. (doi:10.2478/v10039-012-0047-1)

53 Davignon J \& Ganz P. Role of endothelial dysfunction in atherosclerosis. Circulation 2004109 III27-III32. (doi:10.1161/ 01.CIR.0000115644.35804.8B)

54 Taylor PN, Richmond R, Davies N, Sayers A, Stevenson K, Woltersdorf W, Taylor A, Groom A, Northstone K, Ring S et al. Paradoxical relationship between body mass index and thyroid hormone levels: a study using mendelian randomization. Journal of Clinical Endocrinology and Metabolism 2016101 730-738. (doi:10.1210/jc.2015-3505)
Received 8 May 2017

Revised version received 27 June 2017

Accepted 19 July 2017 